\title{
The pleomorphic adenoma of the accessory salivary gland: About a case and review of the literatura
}

\section{Abdellah Dah Cherif, Mohammed Chouche, Younes Barbach, Sara Elloudi, Hanane Baybay, Fatima Zahra Mernissi}

Dermatology Department, University Hospital Hassan II, Fez, Morocco

Corresponding author: Dr. Abdellah Dah Cherif, E-mail: abdellahdahcherif@gmail.com

\begin{abstract}
The pleomorphic adenoma or mixed tumor is defined as a heterogeneous benign tumor of salivary glands. The parotid gland is its principal localisation. It is rare in accessory salivary glands, with a preferential localisation on the oral mucosa. A biopsy may diagnose this tumor but can't eliminate the possibility of another neoplasical location in its center. The anatomopathology examination is mandatory after excision. We report a case of one pleomorph adenoma of the accessory salivary gland is reported in a 56 - year - old woman who underwent surgical resection without recurrence. and review of the literature.
\end{abstract}

Key words: Pleomorphic adenoma; Minor salivary gland

How to cite this article: Dah Cherif A, Chouche M, Barbach $\mathrm{Y}$, Elloudi S, Baybay H, Mernissi FZ. The pleomorphic adenoma of the accessory salivary gland: About a case and review of the literatura. Our Dermatol Online. 2019;10(e):e20.1-e20.4.

Submission: 09.05.2019; Acceptance: 25.05.2019

DOI:10.7241/ourd.2019e.20 


\title{
L' adénome pléomorphe de la glande salivaire accessoire: A propos d'un cas et revue de la littérature
}

\begin{abstract}
Abdellah Dah Cherif, Mohammed Chouche, Younes Barbach, Sara Elloudi, Hanane Baybay, Fatima Zahra Mernissi
\end{abstract}

Dermatology Department, University Hospital Hassan II, Fez, Morocco

Corresponding author: Dr. Abdellah Dah Cherif, E-mail: abdellahdahcherif@gmail.com

\begin{abstract}
RÉSUMÉ
Ladénome pléomorphe anciennement appelé tumeur mixte du fait de sa double composante épithéliale et mésenchymateuse, est une tumeur bénigne au dépend des glandes salivaires qui se développe lentement, à bas bruit, pendant plusieurs années. Ses localisations extra-parotidiennes sont rares.(1) Elles se distinguent de celles a localisation parotidienne par leurs caractéristiques cliniques, histologiques et thérapeutiques.(2) Elles sont réparties entre la glande submandibulaire et les glandes salivaires accessoires (palais, lèvres, tubérosité et joue). Lâge moyen de survenue est de 43 ans avec une prédominance féminine.Lexamen radiologique permet de caractériser la tumeur en évaluant son extension aux tissus avoisinants et de rechercher une lyse osseuse. Le traitement passe par l'exérèse totale de la tumeur. Lexamen anatomopathologique permet de confirmer le diagnostic . (3) On rapporte une localisation rare extra-parotidienne de l'adénome pléomorphe des glandes salivaires accessoires.
\end{abstract}

Mots clés: Adénome Pléomorphe; Glades salivaires accessories

\section{INTRODUCTION}

Ladénome pléomorphe anciennement appelé tumeur mixte du fait de sa double composante épithéliale et mésenchymateuse, est une tumeur bénigne au dépend des glandes salivaires qui se développe lentement, à bas bruit, pendant plusieurs années. Ses localisations extra-parotidiennes sont rares [1]. Elles se distinguent de celles a localisation parotidienne par leurs caractéristiques cliniques, histologiques et thérapeutiques [2].

Elles sont réparties entre la glande submandibulaire et les glandes salivaires accessoires (palais, lèvres, tubérosité et joue). Lâge moyen de survenue est de 43 ans avec une prédominance féminine.Lexamen radiologique permet de caractériser la tumeur en évaluant son extension aux tissus avoisinants et de rechercher une lyse osseuse. Le traitement passe par l'exérèse totale de la tumeur. Lexamen anatomopathologique permet de confirmer le diagnostic [3]. On rapporte une localisation rare extra-parotidienne de l'adénome pléomorphe des glandes salivaires accessoires

\section{CASE REPORT}

Mme MA. S, 56 ans, sans antécédents pathologiques particuliers, a consulté en novembre 2017 pour une gêne au niveau de la lèvre inférieure évoluant depuis 1 an. À l'examen clinique exo-buccal, on note une légère voussure de la lèvre inférieure droite, correspondant à un nodule au niveau de la face interne muqueuse (Fig. 1). Ce nodule est ferme, asymptomatique et mesure $1 \mathrm{~cm}$ de plus grand diamètre. Il est bien délimité, et non fixé, ni sur les plans profonds ni sur la muqueuse labiale, dont l'aspect est semblable à celui de la muqueuse saine. Il n'y avait pas d'adénopathie cervicale ni d'autre masse cervico-faciale palpable. Lexérèse chirurgicale de la tumeur a été réalisée par voie endobuccale, sous anesthésie locale. Lintervention consiste en une simple incision superficielle de la muqueuse de $1 \mathrm{~cm}$ de long, permettant d'extérioriser le nodule par pression

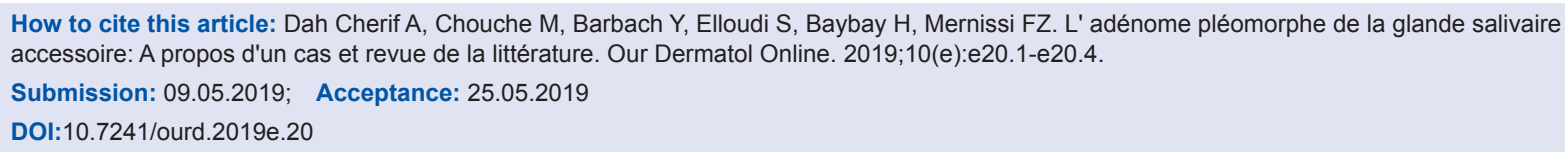


puis de le disséquer facilement des glandes salivaires accessoires avoisinantes. Deux points de suture sont réalisés.Lanalyse histologique précise qu'il s'agit d'une lésion nodulaire bien limitée, correspondant à une prolifération mixte épithéliale et mésenchymateuse avec une fine capsule qui ne présente pas d'effraction. L'examen conclut à la confirmation histologique d'un adénome pléomorphe, encapsulé dont l'exérèse est complète.Â ce jour, 18 mois après, aucune récidive n'a été signalée chez la patiente.

\section{DISCUSSION}

Ladénome pléomorphe est une tumeur bénigne hétérogène au depend des glandes salivaires, longtemps dénommée tumeur mixte du fait de sa double composante épithéliale et mésenchymateuse $[1,3]$.

C'est la tumeur la plus fréquente de la glande parotide $65 \%$. Ses localisations extra-parotidiennes sont rares.

Elles se distinguent de celles a localisations parotidienne par leurs caractéristiques cliniques, histologiques et thérapeutiques. Elles sont réparties entre la glande submandibulaire et les glandes salivaires accessoires [3].

Chez notre patiente, la tumeur siégeait au niveau de glandes salivaires accessoire de la lèvre. D'autres localisations plus rares d'adénome pléomorphe des glandes salivaires accessoires étaient rapportées dans la littérature comme le palais la tubérosité, le septum nasal, le vestibule narinaire, et la base de la langue [1].

Les glandes salivaires accessoires, disséminées dans l'ensemble des voies aérodigestives supérieures, peuvent être le siège de tumeurs rares et réputées bénignes dans la majorité des cas, principalement au niveau de la muqueuse de la bouche (voûte ou voile du palais, plancher de la bouche, joues, lèvres), plus rarement du larynx ou des fosses nasales. Les variétés tumorales sont nombreuses; la plus courante est l'adénome pléomorphe, qui demeure un souci majeur

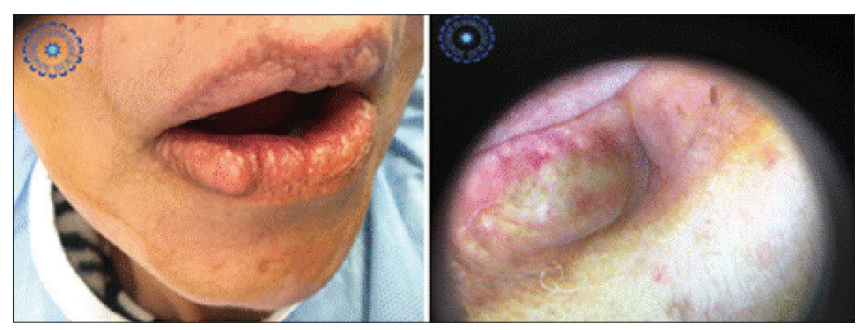

Figure 1: Clinical and dermoscopic photos of the nodule at the level of the lower lip. pour tout médecin dermatologues à cause de son risque de dégénérescence maligne et qui peut se transformer en carcinome après une longue évolution $[3,4]$.

Cette variété est plus fréquente chez la femme avec un âge moyen de survenue de 40 ans comme c' était le cas de notre patiente [4].

Elle siégeait souvent dans les glandes salivaires principales, essentiellement la parotide, mais atteignait également les glandes salivaires accessoires [5].

Quel que soit son siège, l'adénome pléomorphe évolue habituellement selon un mode très lent et asymptomatique.

Chez notre patiente l'adénome était découvert après 1 ans d'évolution, sous la formed'une tuméfaction bien limitée (Fig. 2).

Cependant, des observations d'adénome pléomorphe géant, évoluant sur plusieurs années, ont été décrites notamment au Japon.

Ces formes géantes sont responsables d'un syndrome obstructif des voies aéro-digestives supérieures avec dysphagie, dyspnée, ronflement et rhinolalie. Leur exérèse complète devient délicate et le risque de récidive et de dégénérescence carcinomateuse est plus élevé [5].

La symptomatologie clinique dépendait de la taille et de la localisation tumorale.

Au niveau de la cavité orale, nous décrivons une tuméfaction indolore évoluant sous une muqueuse normale.

Ladénome pléomorphe du palais siégeant le plus souvent en postérolatéral, bridé entre la voûte osseuse et la fibromuqueuse palatine épaisse et saine, la ligne

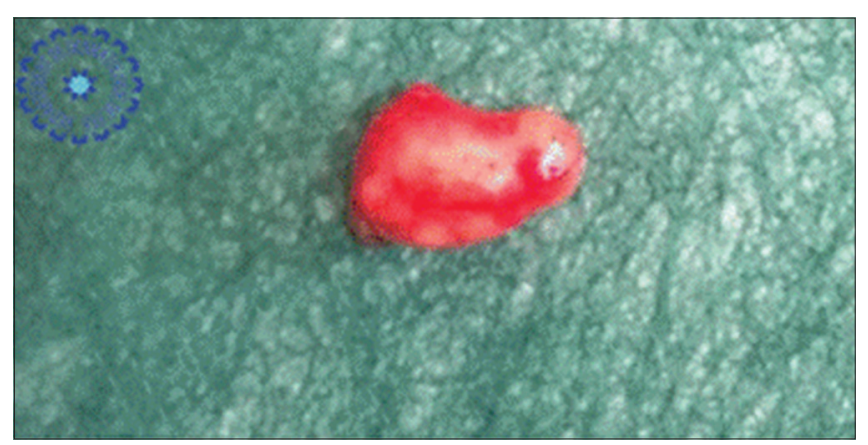

Figure 2: Aspect of the lesion after surgical excision. 
médiane et le voile sont exceptionnellement atteints, contrairement à la tumeur maligne qui déforme la région en "verre de montre».

Ladénome pléomorphe de la joue est une lésion qui se présente sous forme d'un nodule bien limité, ferme et indolore à la palpation, refoulant la muqueuse sans ulcéreration.

Ladénome pléomorphe de la tubérosité se manifeste par un nodule, induré, bien limité et recouvert d'une muqueuse fine Ladénome pléomorphe de la lèvre est une tumeur sous muqueuse de petite taille, indolore, bosselée et recouverte d'une muqueuse normale sans inflammation péri-tumorale [4] comme c'était le cas de notre patiente.

Limagerie est indispensable dans certaines localisation, dans le bilan pré opératoire de l'adénome pléomorphe. Elle repose essentiellement sur la radiographie panoramique, la tomodensitométrie et l'imagerie par résonnance magnétique [6].

Elle permet d'évaluer l'extension de la tumeur par rapport aux tissus avoisinants, de rechercher une lyse osseuse, de mettre en évidence les érosions osseuses et d'établir un bilan d'extension local et locorégional.

Elles est réalisée en cas de lésion volumineuse avant toute intervention chirurgicale.

Histologiquement, l'adénome pléomorphe se caractérise par un polymorphisme cellulaire avec présence de cellules myoépithéliales, épithéliales et stromales, d'où le nom de tumeur mixte $[4,5]$.

Au niveau de la cavité buccale, cette tumeur a pour particularité de ne pas être encapsulée et le contact des cellules tumorales avec les cellules adipeuses ou musculaires ne doit pas en imposer pour un carcinome infiltrant $[4,6]$.

Le traitement est essentiellement chirurgical. Enfin, l'exérèse chirurgicale complète avec excision de la muqueuse de recouvrement et coagulation du lit tumoral fournira le diagnostic définitif et permettra le traitement de cette remarquable tumeur des glandes salivaires. Cette exérèse complète est rendue difficile, quand il s'agit de tumeurs volumineuses, par l'absence de capsule et l'infiltration des glandes salivaire accessoires entre les fibres musculaires et les pannicules adipeux. Elle peut nécessiter le recours à des lambeaux de reconstruction en cas d'excision muqueuse importante [7].

Son pronostic est généralement bon, mais reste marqué par un risque élevé de récidive après chirurgie et de dégénérescence carcinomateuse imposant une prise en charge chirurgicale précoce et une surveillance régulière [8].

\section{CONCLUSION}

L'adénome pléomorphe est une tumeur bénigne hétérogène des glandes salivaires. Ses localisations extra-parotidiennes sont rares [7] et sont dominées par la glande submandibulaire et les glandes salivaires accessoires du palais et des lèvres.

La revue de la littérature confirme le caractère évolutif et invasif en cas d'abstention chirurgicale, et d'autre part, sa tendance à la récidive locorégionale. Lexérèse complète est impérative [8].

La surveillance régulière locorégionale après exérèse doit être systématique pendant plusieurs années

\section{REFERENCES}

1. Kubacka M, Orendorz-Fr-czkowska K, Pazdro-Zastawny K, Morawska Kochman M, Kr-cicki T. Epidemiological evaluation of salivary gland tumors in the Wrocław ENT Department patients in the years 2001-2010. Otolaryngol Pol. 2013;67:30-3.

2. Nitassi S, Oujilal M, Boulaich M, Essakalli L, Kzadri M. Adénome pléomorphe géant de la parotide. Rev Stomatol Chir Max Facial. 2009;110:350-2.

3. Uro-Coste E. Tumeurs des glandes salivaires. État des lieux en 2009. Ann Pathol. 2009;29:274-85.

4. Paris J, Facon F, Chrestian MA, Giovanni A, Zanaret M. Adénome pléomorphe parotidien: Etude Histopathologique, Original Research Article. Ann Otolaryngol Chir Cerv Facial. 2004;121:161-6.

5. Carlson.G. The salivary glands: Embryology, anatomy, and surgical applications. Surg Clin North Am. 2000;80:261-73.

6. Prades JM, Oletski A, Faye MB, Dumollard JM, Timoshenko AP, Veyret C. Morphologie IRM des tuméfactions de la glande parotide. Corrélations histopathologiques, Original Research Article. Morphologie. 2007;91:44-51.

7. Saban Y, Polselli R, Ruvolo V. Chapitre 1 - Anatomie de la face. Chirurgie Plastique Réparatrice De la Face et du Cou - Volume 1, 2011, Pages 3-17.

8. Laskawi R, Schott T, Mirzaie-Petri M, Schroeder M. Surgical management of pleomorphic adenomas of the parotid gland: A followup study of three methods. J Oral Maxillofacial Surg. 1996;54:1176-9.

Copyright by Abdellah Dah Cherif, et al. This is an open access article distributed under the terms of the Creative Commons Attribution License,

which permits unrestricted use, distribution, and reproduction in any medium, provided the original author and source are credited.

Source of Support: Nil, Conflict of Interest: None declared. 